\title{
A TWO DIMENSIONAL SHOCK CAPTURING, HYDRODYNAMIC MODEL OF THE VENUS IONOSPHERE
}

\author{
A. F. Nagy, A Körösmezey1, J. Kim and T. I. Gombosi \\ Space Physics Research Laboratory, The University of Michigan
}

\begin{abstract}
A two-dimensional, time-dependent, shock capturing single-species $\left(\mathrm{O}^{+}\right)$hydrodynamic model of the Venus ionosphere, which solves the coupled continuity, momentum and energy equations for the altitude range of $150-500 \mathrm{~km}$ and the solar zenith angle range of $0^{\circ}-180^{\circ}$ has been developed and is presented in this paper. It was again demonstrated that the introduction of topside heat inflows leads to calculated dayside electron and ion temperatures, which are consistent with the measured values. In order to reproduce the measured electron temperatures, which are roughly constant over all SZA's, the heat inflows had to be reduced significantly over the nightside compared to the dayside values. The calculated transterminator ion flows are supersonic and relatively close to the observed average values. The model predicts a deceleration shock at a SZA of about $135^{\circ}$, consistent with the jon emperature and velocity observations.
\end{abstract}

\section{Introduction}

Our understanding of the controlling physical and chemical processes in the ionosphere of Venus has advanced significantly since the launch of the Pioneer Venus Orbiter (PVO) [Colin, 1980]. The discovery of the transonic flow directed from the dayside toward the nightside of the Venus ionosphere was one of the many surprising facts that PVO established [Knudsen et al., 1980]. The typical high altitude transterminator ion velocities were found to be of the order of $2-3 \mathrm{~km} / \mathrm{s}$ and are supensonic across the terminator at high altitudes. The ion flow is driven predominantly by the large pressure gradient, which is present across the terminator [Knudsen et al., 1981]. The rapid rise in ion temperatures and drop/randomization in ion velocities beyond a zenith angle of about $150^{\circ}$, led Knudsen et al., [1980] to suggest a shock deceleration of the supersonic flow deep on the nightside.

A number of models have been developed to study and establish the mechanisms responsible for these supersonic ion flows, but all of these previous transport models had some limitations (e.g. use of assumed velocity components, limited spatial coverage, lack of shock "capturing" ability). The need for a full-scale, twodimensional, shock capturing model has been obvious for many years. However two dimensional models capable of such calculations are very complex and computer intensive and have only begun to be employed in the field of space plasma physics relatively recently [e.g.Gombosi et al., 1985]. A two-dimensional, timedependent, shock capturing single-species $\left(\mathrm{O}^{+}\right)$hydrodynamic model of the Venus ionosphere, which solves the coupled continuity, momentum and energy equations for the altitude range of $150-500 \mathrm{~km}$ and the solar zenith angle range of $0^{\circ}-180^{\circ}$ has been developed and is presented in this paper. This two-dimensional model will help us to evaluate the importance of supersonic day-tonight flows and, for the first time address, in a quantitative way, the probability of shock formation in the nightside ionosphere of Venus.

\footnotetext{
I on leave from Central Research Institute for Physics, Budapest, 1525, Hungary
}

Copyright 1991 by the American Geophysical Union.

Paper number 91GL00362

$0094-8534 / 91 / 91 \mathrm{GL}-00362 \$ 03.00$

\section{Model Description}

\section{Governing Equations}

The time-dependent two-dimensional continuity, momentum and energy equations for $\mathrm{O}^{+}$ions and electrons are solved selfconsistently. Quasi-neutrality is assumed, i.e. $\mathbf{n}_{\mathbf{i}}=\mathbf{n}_{\mathbf{e}}$, where $\mathbf{n}$ denotes number density. Furthermore, the ion and electrons are assumed to have the same velocity, i.e. uion = uelectron, in other words the current is assumed to be zero. Knudsen et al. [1981] have shown that the transterminator flow is not strongly influenced by the magnetic field; therefore, the use of a hydrodynamic approach, assumed in this model, is sufficient to elucidate the important aspects of the high speed ion flow. The bulk motion of the neutral atmosphere is neglected. This is a reasonable assumption, because, in general, the ion velocities are significantly larger than the neutral ones. The assumption of only a single ion species, $\mathrm{O}^{+}$, was necessary in order to make the numerical solution "feasible". However this is a reasonably good assumption, because above about $190 \mathrm{~km}, \mathrm{O}^{+}$is the dominant ion species and the main aim of these calculations is to establish the ion flows and their effect, which become significant only above about $200 \mathrm{~km}$. The chemical reactions included in the model are listed in Table 1 . In calculating the chemical source for $\mathrm{O}^{+}$from reaction (R1), the $\left[\mathrm{CO}_{2}^{+}\right]$density was approximated from the photochemical equilibrium relationship:

$$
\left[\mathrm{CO}_{2}^{+}\right] \equiv \frac{\mathrm{P}\left(\mathrm{CO}_{2}^{+}\right)}{\left(\mathrm{k}_{1}+\mathrm{k}_{2}\right)[\mathrm{O}]+\mathrm{k}_{3}\left[\mathrm{n}_{\mathrm{e}}\right]}
$$

where $\mathrm{P}\left(\mathrm{CO}_{2}^{+}\right)$is the photoionization rate of $\mathrm{CO}_{2}{ }^{+}$. The continuity equation is:

$$
\frac{\partial \rho}{\partial t}+\frac{1}{A_{r}} \frac{\partial}{\partial r}\left(A_{r} \rho u_{r}\right)+\frac{1}{r A_{\theta}} \frac{\partial}{\partial \theta}\left(A_{\theta} \rho u_{\theta}\right)=S_{c}-S_{1}
$$

The ion momentum equation in the radial direction is:

$$
\begin{aligned}
& \frac{\partial}{\partial t}\left[\rho u_{r}\right]+\frac{1}{A_{r}} \frac{\partial}{\partial r}\left[A_{r}\left(\rho u_{r}^{2}+p_{i}+p_{e}\right)\right]+\frac{1}{r A_{\theta}} \frac{\partial}{\partial \theta}\left[A_{\theta} \rho u_{r} u_{\theta}\right] \\
& =F_{r}^{i}+F_{r}^{e}+u_{r}^{0} S_{c}-u_{r} S_{I}+\frac{\left(p_{i}+p_{e}\right)}{A_{r}} \frac{\partial A_{r}}{\partial r}
\end{aligned}
$$

The ion momentum equation in the angular direction is:

\begin{tabular}{|c|c|c|c|}
\hline & Reaction & & $\begin{array}{c}\text { Rate constant } \\
\left(\mathrm{cm}^{3} \mathrm{sec}^{-1}\right)\end{array}$ \\
\hline (R1) & $\mathrm{CO}_{2}^{+}+\mathrm{O}$ & $\cdots \mathrm{O}^{+}+\mathrm{CO}_{2}$ & $1.0 \times 10^{-10}$ \\
\hline (R2) & $\mathrm{CO}_{2}^{+}+\mathrm{O}$ & $\cdots \mathrm{O}_{2}^{+}+\mathrm{CO}$ & $1.64 \times 10^{-10}$ \\
\hline (R3) & $\mathrm{CO}_{2}++e$ & $\rightarrow C \mathrm{CO}+\mathrm{C}$ & $1.14 \times 10^{-4} / \mathrm{T}_{\mathrm{e}}$ \\
\hline (R4) & $\mathrm{O}^{+}+\mathrm{CO}_{2}$ & $\cdots>\mathrm{O}_{2}{ }^{+}+\mathrm{CO}$ & $9.4 \times 10^{-10}$ \\
\hline (R5) & $\mathrm{O}^{+}+\mathrm{H}$ & $-\rightarrow>\mathrm{H}^{+}+\mathrm{O}$ & $2.5 \times 10^{-11} \mathrm{~T}_{\mathrm{n}}{ }^{1 / 2}$ \\
\hline
\end{tabular}

$$
\begin{aligned}
& \frac{\partial}{\partial t}\left[p u_{\theta}\right]+\frac{1}{A_{r}} \frac{\partial}{\partial r}\left[A_{r} p u_{r} u_{\theta}\right]+\frac{1}{r A_{\theta}} \frac{\partial}{\partial \theta}\left[A_{\theta}\left(p u_{\theta}^{2}+p_{i}+p_{\theta}\right)\right] \\
& =F_{\theta}^{i}+F_{\theta}^{e}+u_{\theta}^{o} S_{c}-u_{\theta} S_{1}+\frac{\left(p_{i}+p_{e}\right)}{r A_{\theta}} \frac{\partial A_{\theta}}{\partial \theta}
\end{aligned}
$$

Table 1. Ion chemical reaction rates 
The ion energy equation is:

$$
\begin{aligned}
& \frac{\partial}{\partial t}\left[\frac{\rho}{2}\left(u_{r}^{2}+u_{\theta}^{2}\right)+\frac{p_{i}}{\gamma_{i}-1}\right]+\frac{1}{A_{r}} \frac{\partial}{\partial r}\left[A_{r} u_{r}\left(\frac{\rho}{2}\left(u_{r}^{2}+u_{\theta}^{2}\right)+\frac{\gamma_{i}}{\gamma_{i}-1} p_{i}\right)\right] \\
& +\frac{1}{r A_{\theta}} \frac{\partial}{\partial \theta}\left[A_{\theta} u_{\theta}\left(\frac{\rho}{2}\left(u_{r}^{2}+u_{\theta}^{2}\right)+\frac{\gamma_{i}}{\gamma_{i}-1} p_{i}\right)\right]+u_{r} \frac{\partial p_{e}}{\partial r}+u_{\theta} \frac{\partial p_{e}}{r \partial \theta} \\
& =Q_{i}+u_{r}\left(F_{r}^{i}+F_{r}^{e}\right)+u_{\theta}\left(F_{\theta}^{i}+F_{\theta}^{e}\right)+\frac{\partial}{\partial r}\left(K_{i} \frac{\partial T_{i}}{\partial r}\right)+\frac{\partial}{r \partial \theta}\left(K_{i} \frac{\partial T_{i}}{r \partial \theta}\right) \\
& +\left(\frac{u_{r}^{02}+u_{\theta}^{02}}{2}+\frac{k_{n}}{\left(\gamma_{i}-1\right) m_{i}}\right) S_{c}-\left(\frac{u_{r}^{2}+u_{\theta}^{2}}{2}+\frac{p_{i}}{\left(\gamma_{i}-1\right) p_{i}}\right) S_{1}
\end{aligned}
$$

The electron energy equation is:

$$
\begin{aligned}
& \frac{\partial}{\partial t}\left[\frac{p_{e}}{\gamma_{e}-1}\right]+\frac{1}{A_{r}} \frac{\partial}{\partial r}\left[A_{r} u_{r} \frac{\gamma_{e}}{\gamma_{e}-1} p_{e}\right]+\frac{1}{r A_{\theta}} \frac{\partial}{\partial \theta}\left[A_{\theta} u_{\theta} \frac{\gamma_{e}}{\gamma_{e}-1} p_{e}\right] \\
& -u_{r} \frac{\partial p_{e}}{\partial r}-u_{\theta} \frac{\partial p_{e}}{r \partial \theta} \\
& =Q_{e}+\frac{\partial}{\partial r}\left(K_{e} \frac{\partial T_{e}}{\partial r}\right)+\frac{\partial}{r \partial \theta}\left(K_{e} \frac{\partial T_{e}}{r \partial \theta}\right)+\frac{k T_{n}}{\left(\gamma_{e}-1\right) m_{e}} S_{c}-\frac{p_{e}}{\left(\gamma_{e}-1\right) \rho_{e}} S_{l}
\end{aligned}
$$

where

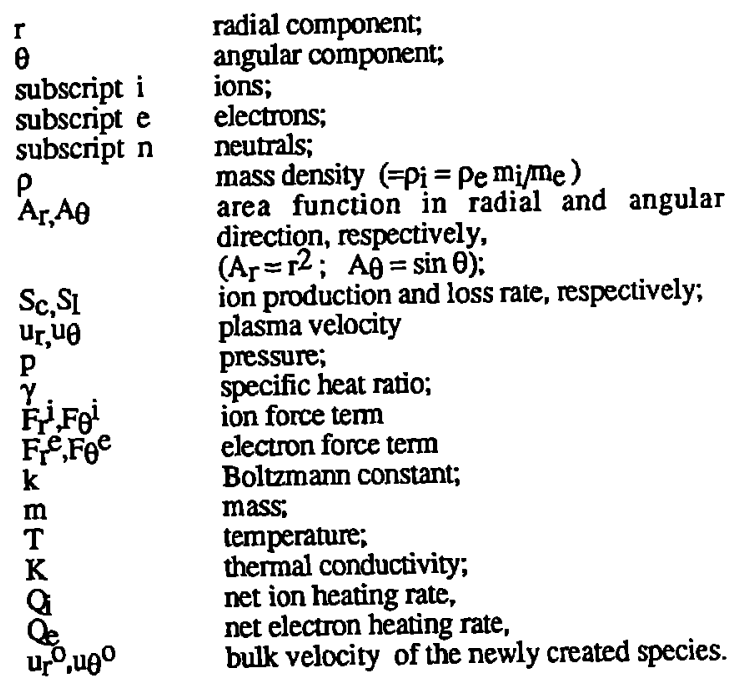

Spherical geometry is used and axial symmetry around the sunVenus axis is assumed. The use of these "five moment" transport equations clearly limits the accuracy of the results obtained from this model. The use of these equations implies that the the temperatures are assumed to be isotropic, the heat flow tensor is replaced by simple thermal conductivities, the flow is inviscid etc; nevertheless this model still gives a good first order description of the major processes controlling the ionospheric dynamics.

\section{Numerical Scheme}

The coupled system of equations (2)-(6) are solved numerically for ion densities $(\rho)$, vertical and horizontal velocities $\left(u_{r}, u \theta\right)$, ion temperatures $\left(T_{i}\right)$ and electron temperatures $\left(T_{e}\right)$. The second order accurate Godunov type scheme, which can handle the shock simulation, is used along with a Crank-Nicholson scheme for handling thermal conduction and heat sources in the energy equations and a Runge-Kutta method is employed for handling other source terms. The Crank-Nicholson scheme is second-order accurate and the Runge-Kutta scheme used is third-order accurate, which in effect give us the overall second order accurate solutions. Similar application of the Godunov type scheme was used by Korosmezey and Gombosi [1989] for their two-dimensional cometary atmosphere model.

\section{Boundary and Initial Conditions}

The upper boundary is set at $500 \mathrm{~km}$ altitude, and the lower boundary is set at $150 \mathrm{~km}$. The two side boundaries are at solar zenith angles (SZA) of $0^{\circ}$ and $180^{\circ}$. The vertical and horizontal resolution is $5 \mathrm{~km}$ and $5^{\circ} \mathrm{SZA}$, respectively. For ion densities $(\rho)$, chemical and diffusive equilibrium conditions are applied at the lower and upper boundaries, respectively. For vertical velocities $\left(u_{r}\right)$, the conditions $u_{\Gamma}=0$ and $\partial u_{r} / \partial r=0$, are imposed at the upper boundary on the day and nightside respectively, but no flows into the system are allowed. Vertical $\left(u_{r}\right)$ and horizontal velocities $(4 \theta)$ are kept equal to zero at the lower boundary. The floating boundary condition, $\partial u_{\theta} / \partial r=0$ is imposed at the upper boundary for the horizontal velocities $\left(u_{\theta}\right)$. For ion and electron temperature $\left(T_{i}, T_{e}\right)$ the heat fluxes, $\phi=-\mathrm{K} \partial \mathrm{T} / \partial \mathrm{r}$, are set at the upper boundary. $\mathrm{T}_{\mathbf{i}}$ and $T_{e}$ are set equal to the neutral temperatures $\left(T_{n}\right)$ at the lower boundary. The floating boundary conditions $\left(\partial \rho / r \partial \theta=0 ; \partial u_{r} / r \partial \theta=0\right.$, $\left.\partial \mathrm{T}_{\mathrm{j}} / \mathrm{r} \partial \theta=0 ; \partial \mathrm{T}_{\mathrm{e}} / \mathrm{r} \partial \theta=0\right)$ are set at the side boundaries $\left(S Z A=0^{\circ}\right.$, $\left.180^{\circ}\right)$, except that the horizontal velocities $(u \theta)$ have reflective boundary conditions ( $u_{\theta, i, n+1}=-u_{\theta, i, n}$ ) at $S Z A=180^{\circ}$.

In order to establish confidence in our model, a number of different test runs were carried out. In one case we dropped all the source terms and perturbed the density to check if the propagation speed is equal to the sonic speed. A test run with all the source terms included was also carried out for the case of no horizontal variations of the input data (photoionization rates, neutral densities, and photoelectron heating rates) to compare with previous onedimensional model results. The results of these pseudo onedimensional calculations are used as the initial conditions for the two-dimensional calculations. A more detailed description of the numerical method can be found in Kim [1991].

\section{Input Parameters}

All input parameters for the model are for solar cycle maximum conditions $\left(F_{10.7}=200\right)$. The neutral densities are from Hedin et al., [1983] except for the $H$ densities, which were taken from VIRA(Venus Intemational Reference Atmosphere) [Keating et al, 1985 ] and extrapolated (VIRA only gives densities for $F_{10.7}=150$ conditions). The presence of hot oxygen is neglected. Photoionization rates and electron heating rates are calculated at every $5^{\circ} \mathrm{SZA}$ and $5 \mathrm{~km}$ altitude grids using the two-stream model of Nagy and Banks [1970]. Ion production rates were arbitrarily decreased from $90^{\circ}$ to $100^{\circ}$ SZA by a factor of 100 and remained decreased from $90^{\circ}$ to $100^{\circ} \mathrm{SZA}$ in order to remove the effect of photoionization on the nightside. On the nightside, the electron heating rates are set to the values at the terminator in order to approximate the heating due to the transported photoelectrons and precipitating energetic electrons.

\section{Results and Discussions}

The computer intensive nature of this model allowed only a limited number of test cases to be run, therefore the input parameters and boundary conditions could not be "tuned for the best" results, in terms of an overall agreement with observations. However the initis use and purpose of this model is to establish the nature of the transterminator ion flow and related energetics in a quantitative and self consistent manner, rather than fit the observed values exactly. Such studies will be carried out in the future, as further computer Such studies will be carried out in the future, as further computer these first calculations (e.g. the presence of hot oxygen atoms) will be included in the work to be presented in a planned, more comprehensive paper. In this short paper we present the results from only one test run, which reproduced the observations in a reasoneble but not exact fashion.

A number of studies showed that heating mechanism(s) other then A number of studies showiation must be considered in the study of the energy balance of the Venus and Mars ionospheres [e.g. Chen o al., 1978; Cravens et al., 1980; Kim et al., 1990]. There have also al., 1978; Cravens et al., 1980; Kim et al., 1990). There he nightside should be smaller than that on the dayside [e.g. Whitten et al. 1986]. For the case presented here constant heat fluxes of $5 \times 10^{9} \mathrm{el}$ $\mathrm{cm}^{-2} \mathrm{~s}^{-1}$ for electrons and $2 \times 10^{7} \mathrm{eV} \mathrm{cm}^{-2} \mathrm{~s}^{-1}$ for ions are imposed at the top boundaries over the dayside and reduced to $30 \%$ of these values on the nightside. The model provides $\mathrm{O}^{+}$densities horizontal and vertical velocities and ion and electron temperaturter these are shown in Figure 1(a)-(d).

The calculated ion temperatures are shown in Figure 1(a). Alto subsolar point, $\mathrm{T}_{i}$ is about $2000^{\circ} \mathrm{K}$, decreases with increasing SZA 
Ion temperature $\left({ }^{\circ} \mathbf{K}\right)$

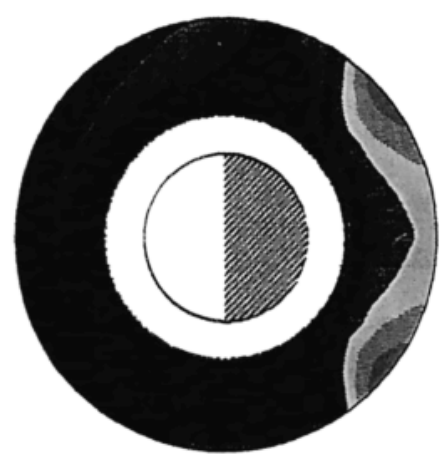

Electron temperature ( $\left.{ }^{\circ} \mathbf{K}\right)$
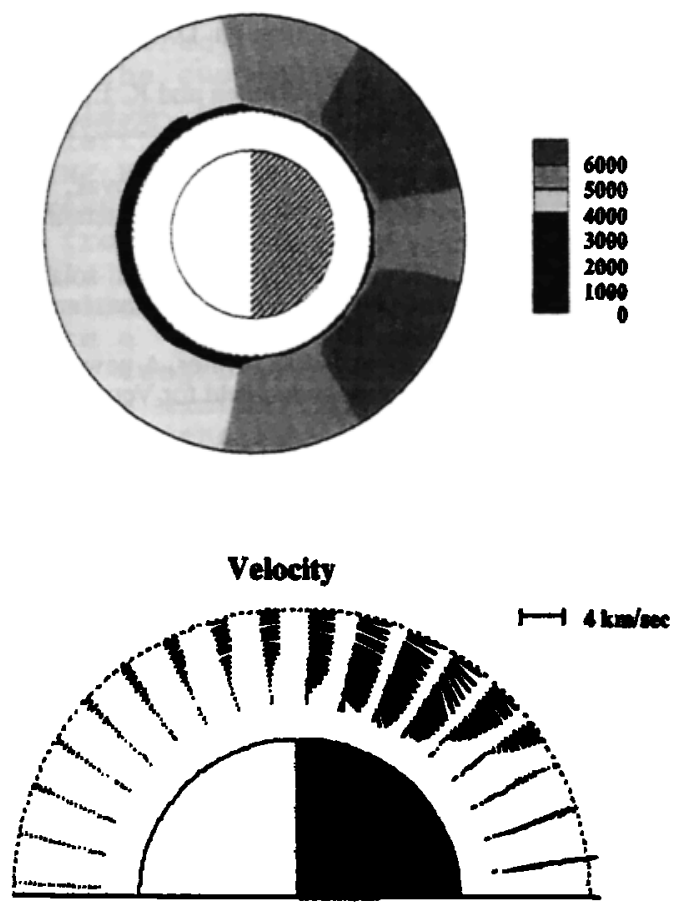

Ion dendittes $\left(\mathrm{cm}^{-3}\right)$

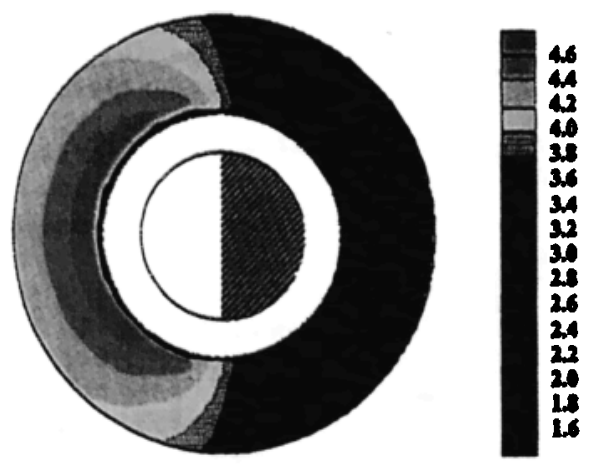

Ion temperature ( $\left.{ }^{\circ} \mathrm{K}\right)$

Fig. 1 (a) Color-shaded contour plot of calculated ion temperatures, (b) plot of calculated electron temperatures, (c) vector plot of calculated velocity fields, and (d) plot of logarithm of calculated ion densities. for $S Z A<100^{\circ}$ and increases with SZA in the nightside, up to $4000^{\circ} \mathrm{K}$ partially due to the convergence of the transonic horizontal flows. There is a drop in the calculated ion temperatures in the region between $90-100^{\circ} \mathrm{SZA}$ as shown in Figure 1(a) due to the adiabatic expansion of the horizontal flow in the region [Knudsen et al., 1980]. Miller et al.[1980] also found some evidence of a slight cooling in the region immediately antisunward of the terminator. There is also a small drop in the ion temperatures past the shock, which is caused by a small increase in the model neutral densities near the antisolar point and adiabatic cooling resulting from the radially outward flow.

The calculated electron temperatures shown in Figure 1(b) are roughily comparable to the measured values, but are somewhat higher on the nightside because the assumed heat inflows are too large for the low density plasma on the nightside. The variation of the electron temperatures are within about $3000^{\circ} \mathrm{K}$ across all SZA range. The median of the observed $T_{e}$ show little variation over all SZÁs [Miller et al., 1980]. In the dayside Venus ionosphere, the two heat sources considered are the topside heat flow and the photoelectron heating. The latter decreases as SZA increases on the dayside and drops sharply after the terminator, unless the effect of energetic electron precipitation is included. Thus the $T_{e}$ would decrease monotonically from the subsolar to antisolar point without an assumed topside heat flow.

The calculated velocity fields shown in Figure 1(c) reach supersonic values up to $3.5 \mathrm{~km} \mathrm{sec}-1$, which are in reasonably good agreement with the measurements [Knudsen et al., 1982]. The horizontal velocities at $397.5 \mathrm{~km}$ are shown in Figure 2 together with the observed values at $\mathbf{4 0 0} \mathrm{km}$ taken from Whitten et al., [1984]. The flow has to be slowed down before it reaches the antisolar region. A shock wave is formed at around $135^{\circ} \mathrm{SZA}$, which can be seen in the vector plot shown in Figure 1(c). Beyond the shock wave, the ion density builds up only slightly [see Figure 1(d)] because horizontally transported ions are lost via recombination. There are strong downward motions on the nightside, with a maximum downward speed of about $600 \mathrm{~m} \mathrm{sec}^{-1}$.

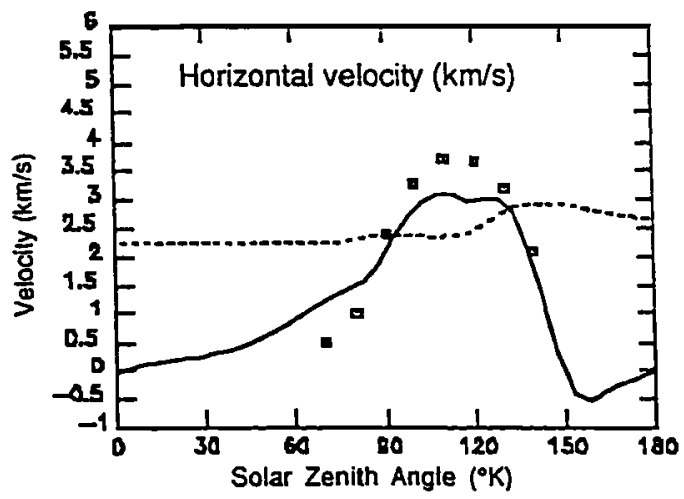

Fig. 2 Calculated horizontal velocities (solid) with sonic speed (dotted) at the altitude of $397.5 \mathrm{~km}$. Squares are the observed horizontal velocities at $400 \mathrm{~km}$ taken from Whitten et al. [1984].

The flow at the top boundaries on the nightside was not prescribed, but was free to float, while the radial flow on the dayside was kept at zero. This top boundary condition for the radial flow, together with the decreasing ion-neutral friction with SZA and mass sink, accelerate the already expanding flow further. Downward motions are dominant in the region between $90^{\circ}$ and $150^{\circ} \mathrm{SZA}$, which can be explained with the stronger $\mathrm{O}^{+}$loss at lower altitudes compared to at higher altitudes, thus forming a relative sink of $\mathrm{O}^{+}$ions at the lower altitudes on nightside. The shock wave front is tilted because the flow stops at lower altitudes earlier than at higher altitudes due to the friction provided by the denser neutral atmosphere at lower altitudes, especially the hydrogen which has a nightside bulge. In a test run without the hydrogen component, the shock wave front was not tilted and was located further away at around $160^{\circ}$ SZA. Beyond the shock wave, there are relatively weaker $\left(-200 \mathrm{~m} \mathrm{sec}^{-1}\right)$ upward motion at all altitudes, allowing the plasma to escape into the tail. 
The calculated ion densities shown in Figure 1(d) decrease with increasing SZA up to about $150^{\circ} \mathrm{SZA}$ and increase slightly with respect to SZA beyond about $150^{\circ} \mathrm{SZA}$. The calculated ion densities above $200 \mathrm{~km}$ drop by about a factor of 600 from $0^{\circ}$ to $150^{\circ} \mathrm{SZA}$. Both the peak $\mathrm{O}^{+}$density and the altitudes of the peak ion density tend to decrease with increasing SZA due to the increasing downward motion from the day to nightside. The photoionization rates of $\mathrm{O}^{+}$and $\mathrm{CO}_{2}{ }^{+}$remain almost constant on dayside and start to drop sharply after $80^{\circ} \mathrm{SZA}$. The ion production rate on the nightside is practically zero. Thus in this model calculation, transport from dayside across the terminator maintains the nightside ionosphere shown in Figure 1(d). Our model includes the loss $\mathrm{O}^{+}$ by charge exchange to $\mathrm{H}$, but not the reverse source reaction. A test nun which eliminated this loss term, resulted in a fourfold increase in the nightside ion densities. Furthermore we use an upper boundary of $500 \mathrm{~km}$; earlier calculations [e.g. Cravens et al., 1983] showed that increasing the effective ionopause altitude leads to higher transterminator fluxes and nighttime densities. Finally it was shown by McCormick et al., [1987] that the nightside densities are very sensitive to the neutral density model used; they could not reproduce the observed densities with the Hedin et al. [1983] model, which was used in this work.

The total height integrated plasma flux across the terminator was evaluated and found to be about $1 \times 10^{26}$ particles sec-1. This value is very nearly equal to the total loss rate integrated over the night side, becaguse the integrated escape flux (which occurs between about $150^{\circ}$ and $180^{\circ}$ SZA) is only about $1 \times 10^{24}$ particles $\mathrm{sec}^{-1}$.

\section{Conclusions}

The first full-scale, self-consistent two-dimensional calculations capable of handling shock waves were carried out for the Venus ionosphere, corresponding to solar cycle maximum conditions. This model successfully simulates the plasma flow over the $0-180^{\circ} \mathrm{SZA}$ range, where traditional numerical scheme could not properly handle the region beyond $150^{\circ} \mathrm{SZA}$

It was again demonstrated that the introduction of topside heat inflows leads to calculated dayside electron and ion temperatures, which are consistent with the measured values. In order to reproduce the measured electron temperatures, which is roughly constant over all SZA's, the heat inflows had to be reduced significantly over the nightside compared to the dayside values.

The model predicts lower ion densities on the nightside than the measured ones; this is believed to be due to 1) the inclusion of the charge exchange loss but not the source reaction, 2) the use of an effective ionopause which is too low and 3) the choice of the neutral atmosphere model. These issues will be addressed in the planned future work, mentioned earlier.

The calculated transterminator ion flows are supersonic and relatively close to the observed average values. The model predicts a deceleration shock at a SZA of about 135 , consistent with the ion temperature and velocity observations.

\section{Acknowledgements}

The work presented in this paper was supported by NASA Grants NAG2-491 and NAGW-1619. Acknowledgement is made to the National Center for Atmospheric Research, which is sponsored by the National Science Foundation, and the San Diego Supercomputer Center for computation resources.

\section{References}

Chen, R.H., Cravens, T.E., and Nagy, A.F., The Martian ionosphere in light of the Viking observations, L. Geophys. Res. 83, 3871, 1978.

Colin, L., The Pioneer Venus program, J. Geophys. Res., 85 $7575,1980$.
Cravens, T.E., Gombosi, T.L., Kozyra, J.U., and Nagy, A.F., Model calculations of the dayside ionosphere of Venus: Energetics, J. Geophys. Res., 85, 7778, 1980.

Cravens, T . E. S. L. Crawford, A. F. Nagy and T. I. Gombosi, A two-dimensional model of the ionosphere of Venus, J. Geophvs. Res. 88, 5595, 1983.

Gombosi, T.I., Cravens, T.E., and Nagy, A.F., A time-dependent theoretical model of the polar wind: Preliminary results, Geophys. Res. Lett., 12, 167, 1985.

Hedin, A.E., Niemann, H.B., Kasprzak, W.T., and Seiff, A., Global empirical model of the Venus thermosphere, $L$ Geophys Res., 88, 73, 1983.

Keating, G.M., Bertaux, J.L., Bougher, S.W., Cravens, T.E, Dickinson, R.E., Hedin, A.E., Krasnapolsky, V.A., Nagy, A.F, Nicholson, J.Y., Paxton, L.J., and Zahn, U.v., Models of Venus neutral upper atmosphere: Structure and composition, Adv. Space Res, 5, 117, 1985 .

Kim, J., A. F. Nagy, T. E. Cravens and H. Shinagawa, Temperature of individual ion species and heating due to charge exchange in the ionosphere of Venus, J. Geophys, Res., 95 , $6569,1990$.

Kim, J., Model studies of the ionosphere of Venus: Ion composition, energetics and dynamics, Ph. D. Thesis, U. of Michigan, 1991.

Knudsen, W. C., K. Spenner, R. C. Whitten and K. L. Miller, Ion energetics in the Venus nightside ionosphere, Geophys. Res. Lett. 7, 1045, 1980.

Knudsen, W.C., Spenner, K., Miller, K.L., and Novak, V., Transport of ionospheric O+ ions across the Venus terminator and implications., J. Geophys Res, 85, 7803, 1980.

Knudsen, W.C., Spenner, K., and Miller, K.L., Anti-solar acceleration of ionospheric plasma across the Venus terminator, Geophys. Res. Lett., 8, 241, 1981.

Knudsen, W. C., P. M. Banks and K. L. Miller, A new concept of plasma motion and planetary magnetic field for Venus, Geophys. Res. Lett., 2, 765, 1982.

Korósmezey, A., and Gombosi, T.I., A time dependent dusty-gas dynamic model of axisymmetric cometary jets, Icarus 84,118 , 1989.

McCormick, P. T., R. C. Whitten and W. C. Knudsen. Dynamics ] of the Venus ionosphere revisited, Icarus, 70, 469, 1987.

Miller, K.L.., Knudsen, W.C., Spenner, K., Whitten, R.C., and Novak, V., Solar zenith angle dependence of ionospheric ion and electron temperatures and density on Venus, J. Geophys. Res., 85, $7759,1980$.

Nagy, A.F., and Banks, P.M., Photoelectron fluxes in the ionosphere, J. Geophys. Res., 75, 6260, 1970.

Whitten, R.C., McCormick, P.T., Merritt, D., Thompson, K.W., Brynsvold, R.R., Eich, C.J., Knudsen, W.C., and Miller, K.L., Dynamics of the Venus ionosphere: A two-dimensional model study, Icarus, 60, 317, 1984.

Whitten, R.C., Singhal, R.P., and Knudsen, W.C., Thermal structure of the Venus ionosphere: A two dimensional model study, Geophys. Res. Lett., 13, 10, 1986.

A. F. Nagy , J. Kim, T. I. Gombosi, Space Physics Research Laboratory, Department of Atmospheric, Oceanic and Space Sciences, The University of Michigan, Ann Arbor, MI 48109 2143.

A. Körösmezey, Central Research Institute for Physics, Budapest, 1525, Hungary.
(Received December 28, 1990; accepted January 24, 1991.) 